\title{
Les risques psychosociaux au travail : d'une " question de société » à des questions scientifiques. Introduction
}

\section{Michel Gollac}

\section{(2) OpenEdition \\ Journals}

Édition électronique

URL : http://journals.openedition.org/travailemploi/5489

DOI : 10.4000/travailemploi.5489

ISSN : $1775-416 \mathrm{X}$

Éditeur

DARES - Ministère du Travail

Édition imprimée

Date de publication : 15 mars 2012

Pagination : $5-10$

ISSN : 0224-4365

Référence électronique

Michel Gollac, «Les risques psychosociaux au travail : d'une « question de société » à des questions scientifiques. Introduction », Travail et Emploi [En ligne], 129 | janvier-mars 2012, mis en ligne le 31

octobre 2012, consulté le 10 décembre 2020. URL : http://journals.openedition.org/travailemploi/5489 ; DOI : https://doi.org/10.4000/travailemploi.5489 


\title{
Les risques psychosociaux au travail : d'une "question de société " à des questions scientifiques Introduction
}

\author{
Michel Gollac(*)
}

Les articles de ce numéro sont, à l'exception de l'étude historique proposée par Nicolas Hatzfeld, issus de travaux réalisés à la demande du Collège d'expertise sur le suivi des risques psychosociaux au travail. À la suite de la conférence sur les conditions de travail d'octobre 2007, le ministre alors en charge du Travail, Monsieur Xavier Bertrand, avait demandé au regretté Philippe Nasse, inspecteur général de l'Insee, vice-président du Conseil de la concurrence et à Monsieur Patrick Légeron, psychiatre, directeur général du cabinet Stimulus, un rapport sur la façon d'identifier, de quantifier et de suivre ces risques. Le «rapport Nasse-Légeron» (NASSE, LÉGERON, 2008) préconisait, entre autres recommandations, la mise en place d'une enquête spécifiquement consacrée aux risques psychosociaux au travail, qui viendrait «[s]'ajouter à l'ensemble des enquêtes existantes, sans supprimer aucune d'elles» et souhaitait que «l'Insee exerce sa responsabilité de garant de la fiabilité sociale de l'enquête en se faisant aider [...] par un Collège d'expertise collective, fonctionnant selon une méthodologie reconnue et validée [...] impliquant en particulier : la pluridisciplinarité, la diversité des points de vue, la traçabilité de leur expression, la qualité scientifique des membres attestée par la liste de leurs publications, une déclaration publique des intérêts qu'ils détiennent». Cette recommandation a été mise en œuvre par le ministre du Travail, qui a demandé à l'Insee de former et d'animer un Collège d'expertise sur le suivi des risques psychosociaux au travail, demande que l'Insee a acceptée. Le Collège ainsi formé a bénéficié de l'appui intellectuel et financier de la Dares et de la Drees. Ses travaux se sont fondés sur l'expertise de ses membres, sur l'audition des partenaires sociaux représentés au Conseil national de l'information statistique, sur celle de nombreuses personnalités scientifiques et sur dix revues de littérature dans les disciplines concernées. C'est donc une commande publique d'expertise qui a débouché sur les articles scientifiques rassemblés ici.

\section{Répondre à une demande sociale}

La qualité et l'originalité des travaux réalisés dans le cadre de ce Collège, et dont le numéro de la revue rend compte, sont dues à la forte mobilisation du monde de la recherche pour répondre à ce qui lui apparaissait comme une demande sociale prioritaire. Les scientifiques sollicités pour faire partie du Collège d'expertise l'avaient été en raison de leur reconnaissance scientifique, qui ne va pas sans une charge de travail en rapport. À quelques exceptions près, liées à des engagements antérieurs, les chercheurs ont accepté de s'engager dans un travail d'ampleur, impliquant les difficultés inhérentes à la coopération entre courants et disciplines. Les scientifiques auditionnés ont dû consacrer du temps non seulement à l'entrevue, mais aussi à sa préparation. Or aucune demande d'audition de chercheurs n'a rencontré de refus. C'est avec le même souci du bien public que d'autres chercheurs ont accepté la lourde charge de participer à la réalisation des revues de littérature.

La question des risques psychosociaux au travail est en effet devenue une question sociale et politique. Très présente aujourd'hui dans les médias (LORIOL, 2011), elle semble retenir l'intérêt de leur public. Elle soulève même une émotion intense lorsqu'elle se présente sous la forme d'événements dramatiques comme les suicides liés au travail. Alors que le travail paraissait promis à la disparition

(*) Centre de recherche en économie et statistique (CREST); michel.gollac@ensae.fr 
en tant que source d'inspiration des écrivains et des artistes (MéDA, 1995), il redevient un sujet possible de films, de téléfilms, de pièces de théâtre, d'ouvrages littéraires dans lesquels il apparaît souvent comme source d'une souffrance liée à ce que nous appelons, dans le jargon scientifique, des facteurs psychosociaux de risque. Le monde politique s'est saisi du problème. Outre le rapport NasseLégeron et celui sur le suivi des risques, des commissions émanant de l'UMP-Nouveau Centre, du Sénat, de l'Assemblée nationale et du Premier ministre ont étudié respectivement la souffrance au travail (COPÉ et al., 2010), le mal-être au travail (DÉRIOT, 2010), les risques psychosociaux au travail et le bien-être au travail (LACHMANN et al., 2010). Le plan santé-travail 2010-2014 accorde quant à lui une importance particulière aux risques psychosociaux. Toutefois, le thème a été peu présent dans les campagnes électorales de 2012.

L'approche en termes de risques psychosociaux a fait l'objet de critiques. L'usage de l'expression «risques psychosociaux au travail» a été promu par l'acteur patronal (MARICHALAR, MARTIN, 2011). «L'expression met en cause un environnement à risques plutôt qu'une personne ou une fonction déterminée, ce qui fait qu'elle se prête moins à l'imputation de responsabilité» (p. 32) sur le mode politique ou juridique. Au contraire, l'invocation de la souffrance au travail renvoie à la psychodynamique du travail, laquelle «fait remonter la cause de la souffrance psychique à l'organisation du travail, et notamment au décalage travail prescrit-travail réel, non pris en compte par les directions d'entreprise. Dans ce cadre théorique, ce sont donc les responsables de la prescription et de l'organisation du travail qui se trouvent mis en cause $[\ldots] »$ (p. 32).

Ces critiques doivent, selon moi, être prises au sérieux. L'idée selon laquelle les directions pourraient faire le bien des travailleurs en s'appropriant le traitement des facteurs de risque isolés a déjà prouvé sa nocivité : par exemple, les réformes managériales des années 1980 et 1990 ont prétendu remédier au manque d'autonomie, mais l'action en ce sens s'est révélée peu efficace et a servi de paravent à une intensification dommageable du travail (BolTANSKI, ChiAPELlo, 1999). Les « risques psychosociaux au travail» figurent en effet plutôt, initialement, dans le vocabulaire patronal, même si les acteurs syndicaux utilisent de plus en plus l'expression : or une situation où l'un des partenaires sociaux jouit d'un monopole sur la définition des situations de travail n'est pas propice à un traitement équilibré, ni même à une connaissance complète de celles-ci. De fait, sous l'étiquette de traitement des risques psychosociaux au travail, certains consultants peu scrupuleux (mais tous ne sont pas dans ce cas !) vendent à des entreprises peu regardantes (mais ce n'est pas le cas de toutes !) des produits inefficaces, voire nuisibles, allant d'enquêtes statistiques de mauvaise qualité à du soutien psychologique bas de gamme.

Faut-il pour autant abandonner l'étude des facteurs psychosociaux de risque au travail? Ce n'est pas mon avis. La diversité des vocables correspond effectivement à la diversité des positions. Mais ceci pourrait bien traduire des effets de concurrence plus que résulter d'une forte cohérence logique entre positions, concepts et vocabulaire, que cette concurrence soit celle entre représentants des salariés et représentants des employeurs ou celle au sein d'un groupe politique ou social. Certes, la «souffrance au travail» fait plutôt partie du répertoire syndical, le patronat utilisant de préférence un autre vocabulaire. Mais elle figurait dans le titre de la commission lancée par l'UMP et le Nouveau Centre et présidée par Jean-François Copé, alors que Xavier Bertrand, ministre en charge du Travail et membre de l'UMP, s'intéressait, on l'a vu, aux «risques psychosociaux». Les travaux de Loriol ont mis en évidence les enjeux complexes et divers de l'emploi de mots comme stress ou burnout (LORIOL, 2003). Par ailleurs, des recherches préliminaires menées par Marichalar tendent à montrer qu'en Suède, le terme «psychosocial» n'est pas porté par les mêmes acteurs politiques et sociaux. Il est d'abord utilisé par des médecins, comme Bertil Gardell ou Töres Theorell, qui cherchent à expliquer des maladies surreprésentées dans certains milieux par d'autres déterminants que l'hérédité ou les comportements individuels, ce qui les amène à s'intéresser au travail (MARICHALAR, 2012). Ces études sur les facteurs de risques collectifs sont mal vues par la droite et les milieux patronaux suédois comme l'illustre en 2006 la suppression de l'Institut national de la vie au travail, jugé trop lié à une pensée sociale-démocrate. Certes, les mots correspondent à des concepts, donc à des théories ou des approches : comme on le verra dans l'article de Pascale Molinier et Anne Flottès, ce ne sont pas les mêmes courants de la psychologie qui parlent de "souffrance» ou de stress. Mais, dans la concurrence des vocabulaires promus par les syndicats et par les directions, c'est cette concurrence qui pourrait bien être le phénomène significatif, le vocabulaire n'en étant qu'un moyen contingent. 
Plutôt que de stigmatiser telle approche comme trop «patronale» ou trop « syndicale», il importe, d'un point de vue scientifique, de préciser la signification accordée à des mots tels que «souffrance», stress ou «risques psychosociaux». En fait, on utilise l'expression de «risques psychosociaux au travail» pour deux choses bien distinctes. Dans un premier usage, elle qualifie des troubles plus ou moins précis de l'état psychique de certains travailleurs. Ces troubles étant, en général, peu spécifiques du travail, le concept est peu utile pour une approche scientifique (il est notamment moins précis que «stress au travail» ou que «souffrance au travail»); il est même source de confusion, bien qu'il puisse servir à des acteurs de terrain (par exemple pour détecter des cas nécessitant une action urgente : même si l'origine des troubles détectés n'est pas le travail, une entreprise peut estimer de son devoir de proposer une aide à un membre de son personnel en situation de détresse psychique). Un autre usage met au contraire l'accent sur les risques spécifiquement liés au travail et engendrés par des mécanismes psychosociaux. Le Collège d'expertise sur le suivi des risques psychosociaux au travail les définit comme «risques pour la santé mentale, physique et sociale, engendrés par les conditions d'emploi et les facteurs organisationnels et relationnels susceptibles d'interagir avec le fonctionnement mental». L'intérêt d'une telle définition est de rassembler les approches de la plupart des recherches menées dans les différentes disciplines, tout en conservant une précision suffisante pour pouvoir être opérationnalisée. Elle évite également de séparer arbitrairement les risques se concrétisant par des troubles mentaux de ceux se concrétisant par des troubles physiques, lorsque les facteurs causaux sont identiques.

Dans l'étude historique qu'il propose dans ce numéro, Nicolas Hatzfeld examine comment, depuis environ deux siècles, a évolué la formulation des risques encourus par les travailleurs et comment ont varié les causes attribuées à ces risques. Tout au long de la période, il note une opposition entre les représentations mettant l'accent sur les effets d'un travail qui ne conviendrait pas aux travailleurs et celles mettant l'accent sur les effets de caractéristiques des travailleurs qui ne conviendraient pas au travail. Ces points de vue sont bien sûr inégalement portés par les acteurs, mais les configurations concrètes varient surtout selon les périodes historiques. Le traitement de ce que nous nommons aujourd'hui risques psychosociaux au travail «peut, selon les variations de l'expertise et des rapports de force, voir présenter [les travailleurs] aussi bien comme objets de menaces que comme porteurs de nuisances » (p. 22). Au début du XIX ${ }^{\mathrm{e}}$ siècle, le courant hygiéniste met en cause à la fois le mode de vie des travailleurs, leurs conditions de vie et les conditions d'emploi et de rémunération, tout en minimisant l'impact spécifique des conditions de travail stricto sensu. Quant aux travailleurs, face aux débuts du machinisme, ils revendiquent le métier avec «la pénibilité qu'elle entraîne» (p. 13). À la Belle Époque, la question du «surmenage» apparaît au premier plan, en lien avec les débuts du taylorisme et d'autres systèmes de réorganisation du travail. La même époque voit les débuts d'une législation sur la santé au travail. À la Libération, les conditions de travail imposées lors de la crise des années 1930 puis de l'Occupation sont vivement dénoncées et cette dénonciation porte sur les conditions locales (le rôle des chefs notamment) qui ont pu aggraver l'effet de la situation globale. Une psychologie du travail soucieuse de la santé des travailleurs se développe alors. Ces développements, mais aussi la généralisation de la «rationalisation» du travail, vont amener les syndicats à se préoccuper de la «fatigue nerveuse», tandis que les entreprises mettent l'accent sur la sélection des travailleurs et que certains milieux médicaux concourent à la naturalisation des troubles. À partir des années 1980, les transformations du management et la dégradation du marché de l'emploi, qui freine les revendications comme les mobilités volontaires, engendrent une «crise du travail». Les progrès de l'épidémiologie répondent à une demande de connaissances émanant d'acteurs en charge de la santé au travail plus diversifiés tandis que ceux en charge de la régulation macro-économique et macro-sociale jouent un rôle accru. La construction européenne vient pour sa part mettre en question les évidences de la santé au travail à la française. L'importance des comités d'hygiène, de sécurité et des conditions de travail (CHSCT) croît, peut-être à cause de la perte d'influence d'autres instances de représentation, alors que l'approche des conditions de travail se médicalise et se technicise.

Le psychologue Yves Clot critique la notion de risques psychosociaux au travail non pas tant en raison de ses promoteurs que de la démarche intellectuelle et pratique qui l'accompagne. Il voit dans l'intérêt pour les risques psychosociaux ce qu'il appelle une «approche hygiéniste» (CLOT, 2010). D'une part, il s'agirait de repérer des facteurs de risque isolés susceptibles de troubler le rapport du travailleur à un travail par ailleurs sain, un peu comme un microbe pathogène trouble le fonctionnement d'un organisme sain. Il s'agirait d'autre part d'éradiquer ces facteurs de risque afin de procurer 
des conditions de travail salubres à des travailleurs supposés subir passivement leur environnement professionnel. À cette approche, Clot oppose une transformation globale du travail, permettant le déploiement par les travailleurs eux-mêmes d'une activité personnelle indépendante dans le cadre de collectifs professionnels jouissant d'une autonomie collective. La critique émise par Clot s'applique à des approches qui réduisent le travail à quelques facteurs de risque ou confondent les indicateurs de la qualité de vie au travail avec le travail lui-même. La quantification est par nature une réduction et il n'est jamais bon qu'elle soit la seule approche : au contraire, les approches qualitatives et quantitatives se renforcent en se confrontant. A fortiori, il est réducteur d'utiliser comme mesure de la qualité de vie au travail des questionnaires conçus pour examiner l'effet délétère d'un nombre très limité de caractéristiques du travail. Certes, des questionnaires tels que ceux de Karasek, Theorell, ou de Siegrist sont fondés sur des modèles assez puissants pour que cet usage détourné soit efficace dans nombre de cas. Mais, dans d'autres cas, il est trompeur. Quoi qu'il en soit, les efforts des épidémiologistes pour concevoir et tester des approches plus complètes, ou moins incomplètes, du travail sont précieux.

\section{Convergences et spécificités des approches disciplinaires}

C'est de tels efforts que rend compte l'article de Ruth Ndjaboué, Michel Vézina et Chantal Brisson. Après avoir rappelé à quel point les modèles de Karasek, Theorell, et de Siegrist sont à la fois indispensables et insuffisants, ils exposent plusieurs classes de nouveaux modèles, leurs principes, leur portée et leurs limites. Le modèle, ou plutôt les modèles de la justice organisationnelle permettent « de mesurer les situations survenues sur le lieu de travail au-delà de l'aspect individuel [...]» (p. 25). Ces modèles de justice organisationnelle sont assez complexes et peuvent se subdiviser en justice relationnelle, procédurale et distributive. Ils sont de plus pertinents : les diverses formes d'injustice organisationnelle créent en effet un risque pour la santé mentale, et cela même si on élimine l'effet du déséquilibre observé au niveau individuel entre exigences du travail, maîtrise de celui-ci et soutien (modèle de Karasek), ou entre effort et récompense (modèle de Siegrist). Le modèle de la justice organisationnelle ne satisfait sans doute pas entièrement les cliniciens de l'activité, mais il prend mieux en compte les aspects d'autonomie collective, au sens le plus large, que les modèles plus anciens. Il commence à être bien validé et, en complément des modèles classiques, contribue à une vision plus riche du travail. Le modèle de la prédictabilité est, en un certain sens, encore plus proche des préoccupations des cliniciens de l'activité puisqu'il pointe les effets délétères sur les travailleurs d'un défaut de maîtrise de l'avenir de l'environnement professionnel lorsque les salariés ne sont pas pleinement acteurs de son évolution. Même si les études utilisant ce modèle sont encore trop rares, elles semblent aller dans le sens d'une validation si bien que l'on peut parler d'un modèle épidémiologique émergent. Quant aux modèles du leadership, ils forment une famille foisonnante, mais les résultats empiriques des quelques études qui les testent sont peu concluants.

Tout comme celui de Ndjaboué, Vézina et Brisson, l'article d'Hélène Sultan-Taïeb et Isabelle Niedhammer montre, à sa manière, l'utilité des recherches épidémiologiques et la nécessité de les approfondir. Ces deux chercheures ont en effet entrepris d'examiner la possibilité de chiffrer le coût du «stress au travail» en termes de santé publique et en termes économiques. Après avoir exposé l'intérêt d'un tel chiffrage, elles en détaillent une méthode, fondée sur le calcul de «fractions attribuables » à ce stress pour divers troubles de santé : quelle est la part des maladies survenues qui ne seraient pas apparues en l'absence du stress? Puis elles dressent une liste des difficultés méthodologiques rencontrées : les modèles épidémiologiques et, plus encore, ceux pour lesquels on dispose d'assez de résultats pour évaluer des fractions attribuables sont loin de couvrir toutes les dimensions pertinentes du travail; les études ne s'intéressent pas à toutes les pathologies; on est obligé d'utiliser des résultats portant sur des populations différentes de la population générale des travailleurs français. Les auteures relèvent des sources de difficultés plus techniques, comme les variations dans la formulation des questionnaires, même s'ils sont censés être standardisés, ou dans le repérage des pathologies. On pourrait ajouter, par exemple, le fait que la plupart des études disponibles étudient l'impact d'une situation de job strain (travail astreignant), opposée à une autre situation, sans proposer d'analyse plus détaillée de l'impact du niveau précis de job demand (exigence du travail ou demande du travail, par anglicisme) et de job latitude (autonomie au travail ou latitude du travail, là 
encore par anglicisme). Enfin, Sultan-Taïeb et Niedhammer discutent de la possibilité de considérer les associations statistiques mises en évidence par les études épidémiologiques comme dues à une véritable causalité. Leur article souligne à juste titre la nécessité d'améliorer les connaissances, en particulier grâce à des questionnaires suffisamment complets, administrés à des cohortes nombreuses faisant l'objet d'un suivi prospectif. Les recherches épidémiologiques de qualité peuvent paraître coûteuses, mais elles sont un prix à payer pour éviter les gaspillages, sans même parler bien sûr des souffrances causées par un niveau élevé de risques psychosociaux au travail.

Les progrès souhaitables des recherches quantitatives ne diminueront pas l'intérêt des études qualitatives. Souvent, pour ne pas dire le plus souvent, l'approche clinique, et plus généralement qualitative, est le moyen privilégié pour comprendre, dégager les causalités, découvrir des phénomènes inattendus. Pascale Molinier et Anne Flottès ont passé en revue les approches cliniques de la santé mentale au travail pratiquées en France depuis les années 1950, en particulier celles issues de la psychodynamique du travail et de la clinique de l'activité. Elles établissent une distinction entre souffrance et stress. Le stress est une réaction biologique bien précise et générale, même si la perception des menaces et des ressources disponibles pour y faire face renvoie aux singularités des individus. La souffrance est un affect, inséparable du sujet et le courant de la psychodynamique du travail pose que le destin de la souffrance au travail (vécu passif, mise à distance par des défenses, subversion en action, voire en plaisir) dépend de la "résonance symbolique» existant, ou pas, "entre le travail et l'histoire personnelle du sujet» (p. 53). Les auteures estiment, sur cette base, «très délicat» de définir des indicateurs de risques psychosociaux. S'il s'agit de mesurer les risques au niveau de chaque individu, «très délicat» est un euphémisme. Mais dans des collectifs pas trop réduits, certains facteurs de risque vont augmenter l'espérance (terme technique qui sonne bizarrement ici) du nombre de troubles mentaux : la nature des troubles dépendra notamment de l'histoire personnelle des travailleurs concernés. Molinier et Flottès explorent ensuite de façon très fine la question de la reconnaissance du travail. Elles montrent comment la psychodynamique du travail a mis en évidence le fait que la reconnaissance ne se limite pas à la reconnaissance symbolique ni même économique, et a établi le caractère matériel de la reconnaissance pratique qui consiste à disposer des moyens de faire son travail avec soin. Les conflits de valeurs ne se réduisent pas aux conflits éthiques, mais concernent également tous les « conflits sur ce qui compte dans le travail pour les sujets» (p. 54) : les auteures proposent par cette définition de dépasser le désaccord entre psychodynamique du travail et clinique de l'activité. Cette dernière, comme le constatera le lecteur, développe des conceptions (instances organisant le travail et les conflits sur le travail notamment) qui, même si leur origine est souvent dans la linguistique, sont probablement particulièrement à même de féconder le dialogue entre psychologues et sociologues. Enfin, Molinier et Flottès décrivent des domaines qui concentrent aujourd'hui l'attention des cliniciens : troubles musculo-squelettiques, pathologies de surcharge, décompensations psychiatriques, addictions, lien entre idéologies défensives de métier et harcèlement moral, violence et agressivité, harcèlement sexuel, travail émotionnel et engagement du corps érotique dans les activités de service, suicides liés au travail. Dans plusieurs de ces domaines, les conceptions de la psychodynamique du travail et celles de la clinique de l'activité s'affrontent et se complètent.

Damien Cartron et Catherine Guaspare organisent leur revue de la littérature sociologique francophone autour de la notion de désajustement. Empruntée à l'étude de Loup Wolff et Xavier Zunigo sur France Télécom (WolfF, Zunigo, 2010), cette notion est dans la filiation indirecte des réflexions de la sociologie bourdieusienne sur l'hystérésis des habitus (BouRdieu, BolTANSKI, 1975). S'ils observent que les travaux sociologiques montrent souvent des acteurs mettant en lien leurs souffrances ou difficultés au travail d'origine psychosociale avec des «décalages», des "écarts», Cartron et Guaspare restent prudents sur le rôle de ces décalages : causes des difficultés dans certains cas, ils peuvent aussi être le révélateur de problèmes dont l'origine est plus profondément enfouie dans les implicites des organisations. Les auteurs dressent un inventaire des désajustements rapportés par la littérature sociologique : ces désajustements évoquent assez souvent des problèmes traités, mais d'une autre manière, par les disciplines connexes. Par exemple, le lecteur pourra mettre certains en relation avec les modèles épidémiologiques les plus classiques : le désajustement entre les moyens et les objectifs est au cœur du modèle de Karasek, celui entre l'investissement dans le travail et la reconnaissance au cœur de celui de Siegrist. Bien sûr, les études sociologiques enrichissent et élargissent beaucoup notre vision de ces modèles. Autre exemple, les désajustements entre la définition du (bon) travail portée par les salariés et les attentes de leur hiérarchie, ou ceux entre tâches à accomplir et éthique 
des travailleurs font écho aux développements de la psychodynamique du travail et de la clinique de l'activité. La sociologie peut ici apporter une vision dynamique, notamment en abordant la genèse des définitions du bon travail ou des conceptions éthiques, au niveau individuel et collectif, permettant une meilleure compréhension des écarts constatés d'une entreprise, d'une profession ou d'un individu à l'autre. Une originalité de l'étude de Cartron et Guaspare est d'observer que les désajustements sont perçus par les travailleurs à travers des phénomènes «microscopiques», des rencontres singulières entre un travailleur déterminé et une évolution particulière du travail.

Le lecteur pressent dès lors qu'une montée en généralité prématurée, imposée purement de l'extérieur par un regard gestionnaire ou expert, tend à inhiber le pouvoir d'agir des travailleurs et, de ce fait, à limiter les possibilités de lutter contre les risques psychosociaux au travail. Une vision globale est nécessaire pour construire les causes communes des situations singulières et pour concevoir des formes d'action qui ne soient pas trop vite détournées de leur but. Mais elle ne peut valoir que si les acteurs concernés, par leur expression et leurs controverses, prennent part à sa construction. Ce numéro de Travail et emploi pourra y contribuer car il rend bien compte de la diversité des approches disciplinaires, des passerelles qui les relient, des débats aussi qui animent la communauté scientifique.

\section{Bibliographie}

Boltanski L., Chiapello E. (1999), Le Nouvel esprit du capitalisme, Paris, NRF.

Bourdieu P., Boltanski L. (1975), «Le titre et le poste : rapports entre le système de production et le système de reproduction», Actes de la recherche en sciences sociales, no 2, pp. 95-107.

Clot Y. (2010), Le travail à cour. Pour en finir avec les risques psychosociaux au travail, Paris, La Découverte.

Copé J.-F., et al., (2010), Rapport final de la Commission de réflexion sur la souffrance au travail composée de députés UMP et Nouveau Centre, et de personnalités qualifiées, document consultable sur le site http:// www.lasouffranceautravail.fr/tl_files/telechargements/ Rapport \%20final-2.pdf

DÉRIOT G. (2010), Rapport d'information fait au nom de la commission des affaires sociales par la mission d'information sur le mal-être au travail, Sénat, no 642, document consultable sur le site http://www.senat.fr/rap/ r09-642-1/r09-642-11.pdf

Lachmann H., et al. (2010), Bien-être et efficacité au travail. Dix propositions pour améliorer la santé psychologique au travail, rapport fait à la demande du Premier ministre; document consultable sur le site http://lesrapports.ladocumentationfrancaise.fr/BRP/ 104000081/0000.pdf
LORIOL M. (2003), «La construction sociale de la fatigue au travail : l'exemple du burnout des infirmières hospitalières », Travail et emploi, no 94, pp. 65-73.

Loriol M. (2011), «Pourquoi tout ce stress?» in Lallement M., et al., «Maux du travail : dégradation, recomposition ou illusion?», Sociologie du travail, vol. 53, no 1, pp. 9-14.

Marichalar P., Martin E. (2011), «Les syndicats et la souffrance», in Lallement M., et al., «Maux du travail : dégradation, recomposition ou illusion? », Sociologie $d u$ travail, vol. 53, no 1, pp. 29-33.

MÉDA D. (1995), Le travail, une valeur en voie de disparition, Paris, Aubier.

NAsSe P., LÉGeron P. (2008), Rapport sur la détermination, la mesure et le suivi des risques psychosociaux au travail, document consultable sur le site: http://lesrapports. ladocumentationfrancaise.fr/BRP/084000156/0000.pdf

Wolff L., Zunigo X. (2010), «Parcours professionnels, expériences du déclassement et risque psychosocial. Les enseignements du questionnaire France Télécom», in Séminaire annuel du CREAPT : le rôle de l'expérience dans les contextes de changement du travail, 10-12 mai 2010. 